\title{
A Two Parameter Four Neutrino Mixing Model with an Exchange Symmetry of the Mass Doublet Neutrinos
}

\author{
E.M. Lipmanov \\ 40 Wallingford Rd. \#272, Brighton, MA 02135, USA
}

\begin{abstract}
In a model with four neutrino mass eigenstates grouping in two narrow doublets the condition of maximal mixing of the doublet neutrino components leads to a simple 4-neutrino mixing matrix which is related to two connected effective two-neutrino mixing patterns. As a result, large values of the neutrino oscillation amplitudes for both the solar and atmospheric neutrino anomalies appear quite naturally if the LSND data are accepted.
\end{abstract}

In the course of the last few years it was gradually shown [1,2] that all the currently available data on neutrino oscillations can be described in a scheme with four neutrino mass states where the four masses are divided into two pairs of close masses separated by a gap of the order of $1 \mathrm{eV}$. The data of the solar, atmospheric, and LSND experiments can be explained if there is neutrino mixing with the following values of the neutrino mass differences [2]:

$$
\begin{aligned}
\Delta m_{1}^{2} & \equiv \Delta m_{\text {solar }}^{2} \sim 10^{-10}(\text { Vac }), \text { or } \sim 10^{-5}(M S W) \mathrm{eV}^{2}, \\
\Delta m_{2}^{2} & \equiv \Delta m_{\text {atm }}^{2} \sim 10^{-3}-10^{-2} \mathrm{eV}^{2}, \\
\Delta m_{12}^{2} & \equiv \Delta m_{L S N D}^{2} \sim 1 \mathrm{eV}^{2} .
\end{aligned}
$$

Only two possible schemes of the neutrino mass spectra are acceptable, (A) and (B), with

$$
\underbrace{\overbrace{m_{1}<m_{1}^{\prime}}^{\text {atm }} \ll \overbrace{m_{2}<m_{2}^{\prime}}^{\text {solar }}}_{L S N D},
$$

and in scheme (B) the positions of the "solar" and "atm" doublet splittings are exchanged.

In the present paper a methodological attempt is made to unify the solar, atmospheric and the LSND data, and also the reactor and accelerator oscillation data, in a simple explicit two parameter 4-neutrino mixing model with two mass doublets and maximum neutrino doublet mixing with only one parameter to adjust (the $\theta_{L S N D}$ angle) for the currently available data. Let us denote by $\nu_{1}, \nu_{1}^{\prime}, \nu_{2}$ and $\nu_{2}^{\prime}$, the four neutrino mass eigenstates. Then the four eigenstates of the exchange symmetry are

$$
\nu_{1,2}^{s, a}=\frac{1}{\sqrt{2}}\left(\nu_{1,2} \pm \nu_{1,2}^{\prime}\right) .
$$

The physical meaning of the Eq. (2) is that the neutrino mass states within the two doublets are mixed maximally, which seems a natural suggestion for the very close neutrino mass states in the weak interactions. The four phenomenological neutrino states - the three weak interaction eigenstates plus one sterile neutrino $\nu_{s}-$ can be expressed in the form:

$$
\begin{aligned}
& \nu_{e}=\nu_{1}^{s} \cos \theta+\nu_{2}^{s} \sin \theta, \\
& \nu_{\mu}=-\nu_{1}^{s} \sin \theta+\nu_{2}^{s} \cos \theta, \\
& \nu_{\tau}=\nu_{1}^{a} \cos \bar{\theta}+\nu_{2}^{a} \sin \bar{\theta} \\
& \nu_{s}=-\nu_{1}^{a} \sin \bar{\theta}+\nu_{2}^{a} \cos \bar{\theta} .
\end{aligned}
$$

Any permutations of the superscripts $s$ and $a$ in the Eqs. (3)-(6) will not change the results if only the $\nu_{e}$ and $\nu_{\mu}$ in the Eqs. (3) and (何) share the same two exchange eigenstates. Note that all found in Ref. [2] criteria for a 4-neutrino two mass doublets mixing scheme to be viable are fulfilled in the explicit model (3)-(6) with $\theta=\theta_{L S N D}$.

The probability of the $\nu_{\mu} \rightarrow \nu_{e}$ oscillations which are relevant to the LSND effect is

$$
\left|\left\langle\nu_{\mu}(0) \mid \nu_{e}(L)\right\rangle\right|^{2}=\sin ^{2} 2 \theta\left[\left\langle\left\langle\sin ^{2}\left(\frac{\Delta m_{12}^{2} L}{4 E}\right)\right\rangle\right\rangle-\frac{1}{4} \sin ^{2}\left(\frac{\Delta m_{1}^{2} L}{4 E}\right)-\frac{1}{4} \sin ^{2}\left(\frac{\Delta m_{2}^{2} L}{4 E}\right)\right],
$$

where $\theta=\theta_{L S N D}$, and the symbol $\langle\langle\rangle$ in the first term denotes the arithmetic mean value of the appropriate four factors related to the four large mass squared differences among the two neutrino mass doublets. 
The notations are

$$
\Delta m_{1,2}^{2}=m_{1,2}^{2}-m_{1,2}^{\prime 2}, \Delta m_{12}^{2} \cong m_{2}^{2}-m_{1}^{2}
$$

$E$ is the initial beam energy and $L$ is the distance from the source. The first term in Eq. (『) describes the LSND effect [3]:

$$
\sin ^{2} 2 \theta \approx 10^{-2}
$$

The second and third terms in Eq. (7) give respectively small $\nu_{e} \rightarrow \nu_{\mu}$ and $\nu_{\mu} \rightarrow \nu_{e}$ contributions to the dominant terms in the Eqs. (10) and (13) for the solar and atmospheric oscillation probabilities.

The probability of the total appearance oscillations of the electron neutrino into all the other neutrino states $\left(\nu_{\mu}+\nu_{\tau}+\nu_{s}\right)$ :

$$
W\left(\nu_{e} \rightarrow \nu_{\mu}, \nu_{\tau}, \nu_{s}\right) \cong \cos ^{4} \theta \sin ^{2}\left(\frac{\Delta m_{1}^{2} L}{4 E}\right)+\sin ^{4} \theta \sin ^{2}\left(\frac{\Delta m_{2}^{2} L}{4 E}\right)+\sin ^{2} 2 \theta \sin ^{2}\left(\frac{\Delta m_{12}^{2} L}{4 E}\right),
$$

where we disregarded the doublet widths in the third term in comparison with the doublet separation. The first term in Eq. (10) is the dominant one; it describes the solar neutrino oscillations with the amplitude value

$$
A_{\text {solar }} \cong \cos ^{4} \theta \cong 1
$$

It does not depend on the angle $\bar{\theta}$ in the model (3)-(6) and is fully determined by the LSND data [3]. Because the $\sin ^{2} \theta_{L S N D}$ is very small, the solar vacuum neutrino disappearance oscillations in the model (3)-(6) are mostly due to the transitions $\nu_{e} \rightarrow \nu_{\tau}+\nu_{s}$

$$
W\left(\nu_{e} \rightarrow \nu_{e}\right) \cong 1-\cos ^{2} \theta\left(\cos ^{2} \bar{\theta}+\sin ^{2} \bar{\theta}\right) \sin ^{2}\left(\frac{\Delta m_{1}^{2} L_{I}}{4 E}\right) \cong \cos ^{2}\left(\frac{\Delta m_{1}^{2} L_{I}}{4 E}\right)
$$

This is just the original still viable Pontecorvo [4] 2-neutrino maximal mixing solution for the solar neutrino deficit (here $L_{I}$ is the Earth-Sun distance). In Eqs. (10), (12), and (13), a special feature of the model (3)-(6) is reflected: the appearance oscillations between the electron and muon neutrinos $\left(\nu_{e}, \nu_{\mu}\right)$, on the one hand, and the tau and sterile neutrinos $\left(\nu_{\tau}, \nu_{s}\right)$ on the other hand, are determined by the "small" doublet mass squared differences $\Delta m_{1}^{2}$ and $\Delta m_{2}^{2}$ with only small corrections from the "large" neutrino mass squared difference $\Delta m_{12}^{2}$ seen in the LSND experiment. This means that the model predicts negative results with $\sin ^{2} 2 \theta_{\mu \tau} \ll \sin ^{2} 2 \theta_{L S N D}$ for the short baseline $\nu_{\mu}$ oscillation experiments such as CHORUS [9].

The probability of the total appearance oscillations of the muon neutrinos into all the other neutrino states $\left(\nu_{e}, \nu_{\tau}, \nu_{s}\right)$ is:

$$
W\left(\nu_{\mu} \rightarrow \nu_{e}, \nu_{\tau}, \nu_{s}\right) \cong \cos ^{4} \theta \sin ^{2}\left(\frac{\Delta m_{2}^{2} L}{4 E}\right)+\sin ^{4} \theta \sin ^{2}\left(\frac{\Delta m_{1}^{2} L}{4 E}\right)+\sin ^{2} 2 \theta \sin ^{2}\left(\frac{\Delta m_{12}^{2} L}{4 E}\right) .
$$

The first term in Eq. (13) is the dominant one, and describes the atmospheric neutrino oscillations with the amplitude value

$$
A_{\text {atm }} \cong A_{\text {solar }} \cong 1
$$

From the Eqs. (7), (10), (13) and the LSND data it follows that in the model (3)-(6) the solar and atmospheric neutrino deficits are dominated by the transitions of the electron and muon neutrinos into the tau and sterile neutrinos in a complementary way: if the solar $\nu_{e}$ disappearance is mostly $\nu_{e} \rightarrow \nu_{\tau}$ dependent (with $\bar{\theta}<\pi / 4$ ), then the atmospheric $\nu_{\mu}$ disappearance is mostly $\nu_{\mu} \rightarrow \nu_{s}$ dependent, and vice versa (with $\bar{\theta}>\pi / 4$ ). The second possibility is preferred by the latest Super-Kamiokande data [5] which favor the appearance $\nu_{\mu} \rightarrow \nu_{\tau}$ oscillations with large amplitude $\sin ^{2} 2 \theta \simeq 1$ as a better match to the atmospheric $\nu_{\mu}$ deficit data.

From the Eq. (10) it follows that the deviation from unity of the $\nu_{e} \rightarrow \nu_{e}$ survival probability is $\operatorname{small} \lesssim \sin ^{2} 2 \theta_{L S N D}$, for oscillation distances $L \ll 4 \pi E / \Delta m_{1}^{2}$. This is in good agreement with the $\nu_{e}$ disappearance oscillation data such as Bugey, and the appearance oscillation $\nu_{\mu} \rightarrow \nu_{e}$ data from BNL E776 experiment [6], and also with the long-baseline $(L=1 \mathrm{~km})$ reactor $\nu_{e}$-disappearance $\mathrm{CHOOZ}$ experiment [7] where this restriction on the distances is fulfilled quite well. 
From the Eq. (13) it follows that the deviation from unity of the $\nu_{\mu} \rightarrow \nu_{\mu}$ survival probability will be small only at distances $L \ll 4 \pi E / \Delta m_{2}^{2}$, and so a discovery of a large $E / L$ dependent $\nu_{\mu}$-disappearance could be within the reach of the coming long baseline $\nu_{\mu}$ oscillation experiments.

With regard to the solar $\nu_{e}$-disappearance data, the large oscillation amplitude (11) is in disagreement with the small angle MSW solution, but not so with the other two solutions: the large angle MSW solution and the vacuum oscillation solution which are compatible with large appearance oscillation amplitudes (see the discussion in Ref. [8]).

In conclusion, a methodologically simple explicit minimal parameters 4-neutrino mixing model in a two mass doublet scheme with maximal doublet neutrino mixing is considered. The main results are: 1) the equality Eq. (14) of the naturally large solar and atmospheric dominant disappearance oscillation amplitudes; 2) the prevailing contributions of the $\nu_{e} \rightarrow \nu_{\tau}+\nu_{s}$ and $\nu_{\mu} \rightarrow \nu_{\tau}+\nu_{s}$ transitions in the solar and atmospheric appearance oscillations in a complementary way; 3 ) the model unifies naturally the three currently available positive neutrino oscillation data from the solar and atmospheric neutrino deficits and the LSND experiment, plus the negative reactor and accelerator oscillation data, with only one parameter to adjust - the $\theta=\theta_{L S N D}$ angle; 4) the neutrino doublet exchange symmetry seems methodologically useful; maximal mixing in the weak interactions is an attractive suggestion for very close neutrino states in the mass doublets; 5) The present model appears to be in a remarkable agreement with the positive data on atmospheric $\nu_{\mu}$ oscillations reported by the Super-Kamiokande group [10]. As I learned after this work was finished, the results here which are relevant to the Super-Kamiokande data are in good agreement with the those obtained in a recent comprehensive study of the neutrino data in Ref. 11]. The approach here is different, however, it is deductive instead: one physical hypothesis - maximal neutrino doublet mixing in a four-neutrino model - leads necessarily to all the predictions if the LSND data are accepted.

I thank E. Kearns for a preprint of his paper.

[1] D. O. Caldwell and R. N. Mohapatra, Phys. Rev. D48 (1993) 3259; J. T. Peltoniemi and J. W. F. Valle, Nucl. Phys. B406 (1993) 409; Z. Berezhiani and R. N. Mohapatra, Phys. Rev. D52 (1995) 6607; J. R. Primack et al., Phys. Rev. Lett. 74 (1995) 2160; E. Ma and P. Roy, Phys. Rev. D52 (1995) R4780; R. Foot, and R. R. Volkas, Phys. Rev. D52 (1995) 6595; E. J. Chun et al., Phys. Lett. B357 (1995) 608; J. J. Gomez-Cadenas and M. C. Gonzales-Garcia, Z. Phys. C71 (1996) 443; S. Goswami, Phys. Rev. D55 (1997) 2931; A. Yu. Smirnov and M. Tanimoto, Phys. Rev. D55 (1997) 1665; E. Ma, Mod. Phys. Lett. A11 (1996) 1893.

[2] S. M. Bilenky, C. Giunti and W. Grimus, Eur. Phys. J., C1 (1998) 247; S. M. Bilenky et al., Phys. Rev. D57 (1998) 1920 ; N. Okada and O. Yosuda, TMUP-HEL-9605, hep-ph/9606411.

[3] C. Athanassopoulos, et al. Phys. Rev. Lett. 77 (1996) 3082.

[4] B. Pontecorvo, Zh. Eksp. Teor. Fiz. 33 (1957) 549; S. L. Glashow and L. Krauss, Phys. Lett. B190 (1988) 199; V. Barger R. J. N. Phillips and K. Whisnant, Phys. Rev. D43 (1991) 1110, and Phys. Rev. Let. 69 (1992) 3135; A. Acker, S. Pakvasa and J. Pantaleone, Phys. Rev. D43 (1991) 1754; P. I. Krastev and S. T. Petcov, Phys. Rev. Lett. 72 (1994) 1960;

[5] E. Kearns, Int. Conf. On Solar Neutrinos, Santa Barbara, CA 1997; TAUP 97, the 5th Int. Workshop on Topic in Astroparticle and Underground Physics, Sept. 1997, Grand Sasso, Assergi, Italy; Y. Totsuka, in Prec. of the Lepton Photon Conf., Hamburg, Germany 1997.

[6] Bugey Coll., B. Achkar et al., Nucl. Phys. B434 (1995) 503; BNL E776, L. Borodovsky, et al. Phys. Rev. Lett, 68 (1992) 274.

[7] CHOOZ Coll., M. Apollonio et al., hep-ex/9711002.

[8] P. I. Krastev and S.T.Petcov, Phys. Lett. 299 (1993) 99; N. Hata and P. G. Langacker, Phys. Rev. D50 (1994) 632 ; J. N. Bahcall and P. I. Krastev, Phys. Rev. D53 (1996) 4211.

[9] CHORUS Coll., E. Eskut et al., Phys. Lett. B424 (1998) 202.

[10] T. Kajita, presented at the XVIII ${ }^{\text {th }}$ International Conference on Neutrino Physics and Astrophysics, Takayama, Japan, June 1998.

[11] V. Barger, S. Pakvasa, T. J. Weller and K. Whisnant, hep-ph/9806328. 